\title{
Estudo Experimental das Características do Escoamento em Escadas para Peixes do Tipo Ranhura Vertical - Padrões Gerais do Escoamento
}

\author{
Daniela Guzzon Sanagiotto \\ Departamento de Engenharia Sanitária e Ambiental - UFSM \\ dsanagiotto@gmail.com \\ António Nascimento Pinheiro \\ Departamento de Engenharia Civil, Instituto Superior Técnico - Lisboa, Portugal \\ apinheiro@civil.ist.utl.pt \\ Luiz Augusto Magalhães Endres, Marcelo Giulian Marques \\ Instituto de Pesquisas Hidráulicas - UFRGS \\ endres@ufrgs.br,mmarques@iph.ufrgs.br \\ Recebido: 08/04/11 - revisado: 20/05/11 - aceito: 26/09/11
}

\section{RESUMO}

A construção de barramentos ao longo dos rios causa muitas alterações no meio envolvido. Entre esses impactos, tem-se a formação de uma barreira que impede o deslocamento do habitat aquático, interferindo, principalmente, na reprodução das espécies migratórias. Na tentativa de mitigar esse efeito, em muitas barragens, verifica-se a necessidade da implantação de mecanismos de transposição de peixes (MTP). Entre esses MTP têm-se as escadas para peixes que, quando empregadas e projetadas adequadamente, possibilitam a livre circulação das espécies. As escadas/passagens para peixes apresentam diferentes geometrias, de acordo com características natatórias dos peixes, altura do obstáculo e vazões disponíveis. Neste trabalho, avaliam-se as características do escoamento em uma escada do tipo ranhura vertical. Realizaram-se ensaios em um modelo com 0,60 m de largura, 0,60 m de profundidade, $9 \mathrm{~m}$ de comprimento e $6 \%$ de declividade, onde se representa, na escala 1:5, parte da escada para peixes instalada na UHE Igarapava (Minas Gerais). Foram realizadas medições da profundidade do escoamento, das pressões junto ao fundo e das velocidades do escoamento, em uma das bacias da estrutura, para três descargas. A região da ranhura na bacia onde ocorre a passagem da água entre bacias consecutivas foi estudada detalhadamente, já que representa uma região de passagem obrigatória e onde se esperam as maiores velocidades. Os resultados deste trabalho foram divididos em duas partes: (1) os parâmetros médios do escoamento e (2) características turbulentas do escoamento. Neste artigo, que representa a parte 1, são apresentados parâmetros adimensionais do escoamento e mostramse através de campos de isolinhas as características da profundidade do escoamento, das pressões e das velocidades médias do escoamento. Os resultados permitem caracterizar o comportamento médio do escoamento nessa estrutura e definir adimensionais importantes no dimensionamento dessas estruturas, entre eles, o coeficiente de descarga e a vazão adimensional. Quanto às velocidades do escoamento, verifica-se que estas não se alteram em função da descarga e da profundidade do escoamento, sendo a posição na bacia o principal fator que interfere. As velocidades máximas chegam, transpondo para o protótipo, a 2,0 $\mathrm{m} / \mathrm{s}$, sendo que predominam as componentes horizontais. As componentes verticais não ultrapassam $0,7 \mathrm{~m} / \mathrm{s}$ (no protótipo) em regiões isoladas. Uma análise que confrontou os campos de velocidade na estrutura e as velocidades características de algumas espécies presentes na ictiofauna brasileira demonstrou que há regiões do escoamento que podem representar barreiras ao livre deslocamento dessas espécies.

Palavras-chave: escadas para peixes, mecanismos de transposição de peixes, velocidades, parâmetros hidráulicos, dissipação da energia.

\section{INTRODUÇÃ̃O}

Cada parte de um rio constitui o habitat de determinadas espécies de peixe, que aí se estabele- ceram devido às características do local: velocidade da corrente, profundidade da água, natureza do leito e das margens, possibilidade de movimentação, regime do rio e qualidade da água. Qualquer mudança nessas características provoca uma alteração 
ou redistribuição da população de peixes (CBDB, 1999).

Por esses motivos, durante a fase de planejamento de um barramento deve-se estudar a ictiofauna local, buscando conhecer as suas características reprodutivas, alimentares, natatórias e migratórias, para que sejam tomadas medidas no sentido da conservação da vida aquática.

As medidas para atenuação do impacto no meio aquático incluem a implantação ou planejamento de mecanismos de transposição de peixes (MTP) para a operação durante toda a vida útil do barramento. Os mecanismos de transposição de peixes são estruturas ou sistemas que possibilitam a migração da ictiofauna entre as partes de jusante e montante de uma barragem, sendo muito importantes, principalmente, por permitir a reprodução dos peixes de piracema que se deslocam em direção às cabeceiras dos rios nesse processo. Os MTP, de uma forma geral, podem ser escadas/passagens para peixes, eclusas, elevadores ou sistemas de captura, transporte e soltura.

Muitos dos MTP apresentam projetos inadequados, com base em critérios internacionais, que não consideram parâmetros biológicos locais, por falta de informações da ictiofauna, por exemplo, e/ou são operados incorretamente, resultando em dispositivos ineficientes.

O conhecimento da capacidade natatória dos peixes e das condições hidráulicas das estruturas é essencial para o desenvolvimento de critérios de projeto adequados para escadas/passagens para peixes. Além das velocidades médias, as características da turbulência do escoamento devem ser avaliadas. Acredita-se que existem níveis ótimos de turbulência para os peixes migratórios (Odeh et al., 2002). Lupandin (2005) estudou o efeito da turbulência no comportamento natatório da espécie Perca fluviatilis, concluindo que a turbulência pode diminuir os índices de locomoção, afetando com maior severidade os indivíduos menores.

Neste contexto, verifica-se a necessidade de novos estudos em modelos de MTP, através da caracterização hidráulica, avaliando além das componentes médias do escoamento, os parâmetros da turbulência, buscando subsídios para relacionar esses fatores com as preferências biológicas.

\section{BREVE REVISÃO BIBLIOGRÁFICA}

$\mathrm{Na}$ sequência apresentam-se algumas informações sobre as passagens/escadas para peixes do tipo ranhura vertical, remetendo-se aos principais trabalhos encontrados na literatura relacionados à caracterização hidráulica do escoamento.

\section{Escadas para Peixes do Tipo Ranhura Vertical}

A primeira escada para peixes do tipo ranhura vertical foi construída em 1943, na Hell's Gate, no Rio Fraser, Canadá (Clay, 1995). Uma escada para peixes do tipo ranhura vertical consiste em um canal retangular, inclinado, dividido por defletores formando várias bacias (Rajaratnam et al., 1986). A água escoa de uma bacia a outra passando por ranhuras verticais. O escoamento forma um jato na ranhura e a energia deste é dissipada pela mistura da água na bacia (Wu et al., 1999), como pode ser observado no esquema da Figura 1a. Geralmente há duas regiões de recirculação, adjacentes ao jato principal, onde se formam zonas de descanso. Segundo Clay (1995), a velocidade da água nas ranhuras deve ser inferior à velocidade de explosão das espécies e a velocidade na bacia deve ser menor que a velocidade de cruzeiro. No entanto, essas proposições têm sido discutidas, sendo que a maioria dos pesquisadores considera que a utilização da velocidade de explosão na passagem pelas ranhuras levaria os indivíduos à fadiga ao longo do percurso.

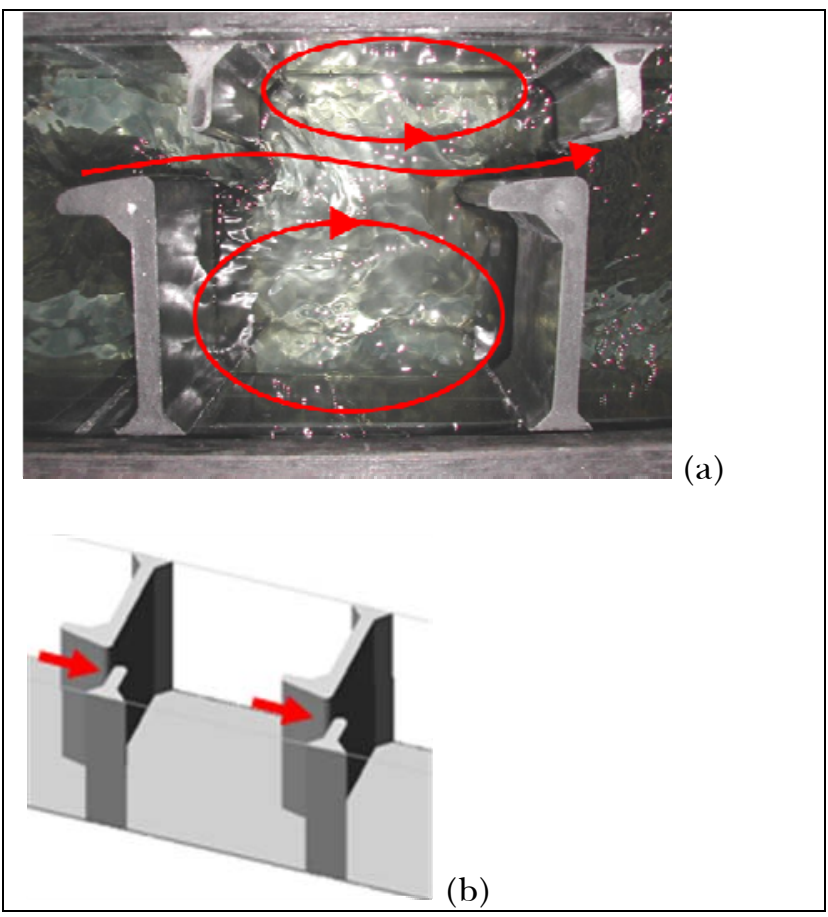

Figura 1 - Escada para peixes com ranhuras verticais: (a) Vista de uma bacia com as linhas de fluxo principais e (b) Esquema de uma escada para peixes do tipo ranhura vertical. 
As escadas para peixes com ranhuras verticais são mais adequadas para peixes que nadam na corrente (não saltadores), pois estas apresentam a capacidade de adaptação às variações de nível maior do que as do tipo soleira vertedoura. Essas estruturas podem apresentar diferentes tamanhos e disposição das ranhuras, dependendo da capacidade natatória dos peixes que irão utilizá-la. A Figura 1b apresenta a configuração geral de uma escada para peixes do tipo ranhura vertical. A definição da geometria desses mecanismos deve ser realizada através de estudos hidráulicos considerando a ictiofauna do local de implantação.

\section{Parâmetros Hidráulicos}

Na sequência serão apresentados alguns parâmetros adimensionais que geralmente são úteis na fase de dimensionamento das escadas para peixes.

\section{Coeficiente de Descarga}

O coeficiente de descarga $(\mathrm{Cd})$ pode ser obtido pela equação (1):

$$
C_{d}=\frac{Q}{b_{0} \bar{y} \sqrt{2 g \Delta h}}
$$

sendo: $Q$ a vazão; $b_{0}$ a largura da abertura entre os septos; $\bar{y}$ a profundidade do escoamento na parte de montante da ranhura; $g$ a aceleração da gravidade e $\Delta h$ a perda de energia entre duas bacias consecutivas, podendo ser dada pelo desnível entre bacias consecutivas, desprezando-se a diferença entre as energias cinéticas.

A suavização das formas da ranhura a montante aumenta o coeficiente de descarga dessas estruturas. Segundo Larinier (2002), esse valor varia entre 0,65 e 0,85 .

\section{Vazão Adimensional}

A vazão adimensional $\left(Q^{*}\right)$ pode ser obtida através da equação (2):

$$
Q^{*}=\frac{Q}{\sqrt{g S_{0} b_{0}^{5}}}=\frac{h_{m}}{b_{0}} \sqrt{\frac{2}{m C_{f}}}
$$

sendo: $\mathrm{h}_{\mathrm{m}}$ a profundidade média do escoamento; $\mathrm{S}_{0}$ a declividade do canal; $\mathrm{C}_{\mathrm{f}} \mathrm{o}$ coeficiente de cisalhamento; $m$ um coeficiente que depende da geometria da ranhura (neste caso $m=2$, porque há fluido contornando o jato principal dos dois lados).

\section{Coeficiente de Cisalhamento}

O coeficiente de cisalhamento $\left(\mathrm{C}_{\mathrm{f}}\right)$ pode ser obtido através da Eq. (2).

\section{Dissipação da Energia}

Bell (1973) apud Bell (1990) propôs, pela primeira vez, a associação de um critério para definir o máximo escoamento em uma passagem para peixes. Bell sugeriu que a máxima dissipação de energia dentro da bacia deveria ser de $0,191 \mathrm{~kW} / \mathrm{m}^{3}$. Muitos autores utilizam esse parâmetro como indicador da turbulência do escoamento, inclusive associando determinadas faixas de potência dissipada por unidade de volume a certos tipos de peixes.

A potência dissipada por unidade de volume $\left(\mathrm{P}_{\mathrm{V}}\right)$ na bacia pode ser obtida pela Eq. (3):

$P_{V}=\frac{Q \cdot \gamma \cdot \Delta h}{\text { volume do tanque }}=\frac{Q \cdot \gamma \cdot \Delta h}{B \cdot L \cdot h_{m}}$

onde: $Q$ é a vazão; $\gamma$ o peso específico da água; $\Delta h$ o desnível entre piscinas; $B$ a largura da bacia; $L$ o comprimento da bacia e $h_{m}$ a profundidade média do escoamento.

\section{Profundidade do Escoamento}

A profundidade do escoamento dentro da bacia é responsável por garantir o conforto para o peixe ao longo da transposição. Valores mínimos devem ser observados para que os possíveis obstáculos dentro da escada, como pedras, não criem regiões com velocidades de fluxo muito elevadas e/ou situações com elevada aeração.

\section{Velocidades}

A velocidade do escoamento é um dos parâmetros mais importantes de uma escada para peixes. Dentro da estrutura devem existir regiões de descanso (baixas velocidades) e, ainda, deve haver um fluxo suficientemente atrativo para o peixe, indicando o caminho a seguir. No entanto, as velocidades máximas devem obedecer a certos limites, relacionados à capacidade natatória dos peixes, para assegurar que seja possível a transposição pela escada, sem que ocorra a fadiga excessiva dos indivíduos. 


\section{INSTALAÇÃO EXPERIMENTAL E MÉTODOS}

\section{Descrição da estrutura}

O trabalho experimental foi realizado no Instituto de Pesquisas Hidráulicas da Universidade Federal do Rio Grande do Sul e reproduz 9 bacias da escada para peixes da UHE de Igarapava na escala geométrica 1:5 (Figura 2).

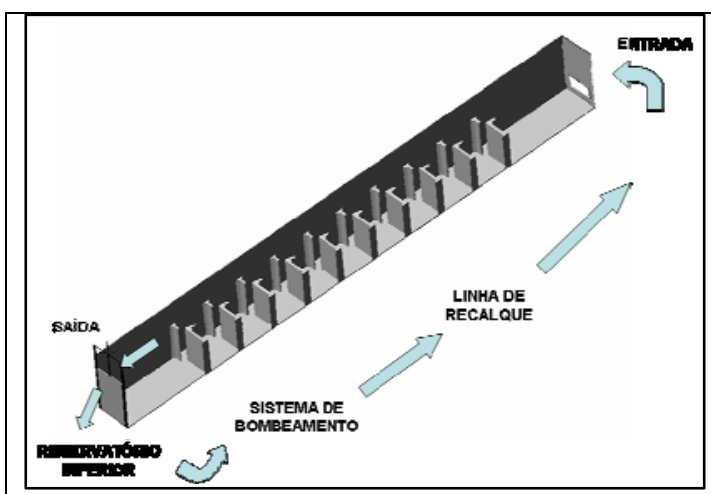

(a)

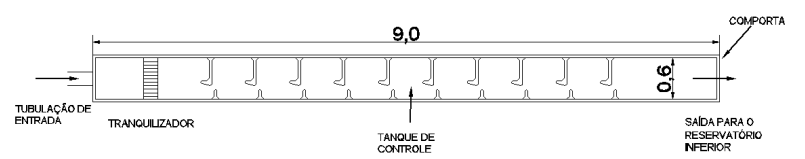

(b)

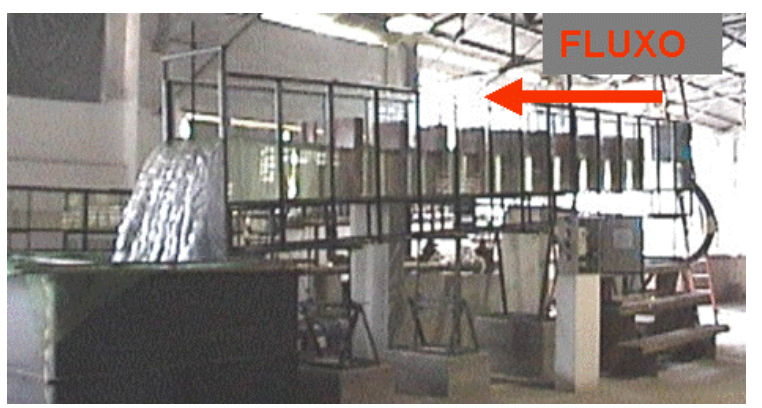

(c)

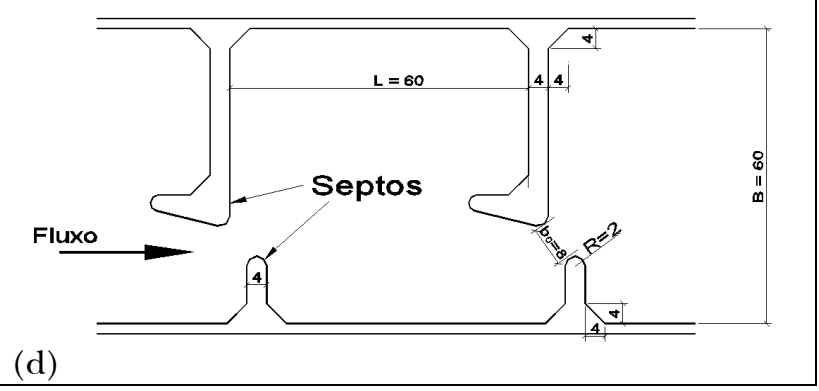

Figura 2 - Modelo reduzido utilizado no estudo: (a) Esquema geral; (b) Planta baixa; (c) Imagem das instalações;

(d) Detalhes da estrutura (dimensões em centímetros).
A estrutura do modelo é composta por um canal com declividade do fundo $\left(\mathrm{S}_{0}\right)$ igual a $6 \%$, com aproximadamente $9,0 \mathrm{~m}$ de comprimento e $0,60 \mathrm{~m}$ de largura (Figuras 2a e 2b). As bacias possuem 0,60 $\mathrm{m}$ de largura (B) e 0,60 $\mathrm{m}$ de comprimento (L). A Figura 2c apresenta uma vista geral do sistema. A passagem da água entre as bacias consecutivas ocorre por uma ranhura vertical com uma abertura $\left(b_{0}\right)$ de $0,08 \mathrm{~m}$ entre um septo maior e outro menor (Figura 2d).

O canal é alimentado por uma tubulação que, com o auxílio de uma bomba operada em conjunto com um inversor de frequência, leva a água de um reservatório inferior até a entrada do canal (parte mais elevada). Desde a parte de entrada da água no canal até o primeiro septo há uma distância de $2 \mathrm{~m}$. Ao longo desse trecho há um tranquilizador com 0,20 m de comprimento, formado por tubos de PVC de $50 \mathrm{~mm}$ de diâmetro, dispostos em forma de colmeia ao longo de toda seção transversal do canal. Após o último septo, tem-se uma distância de 1,50 m até a comporta no final do canal, que verte água para o reservatório inferior. A comporta foi usada para regular o nível de jusante de modo a garantir vazão e lâmina d'água semelhantes às encontradas no protótipo na bacia de controle.

\section{Equipamentos e Metodologia}

Neste trabalho foram realizadas medições da profundidade do escoamento, de pressões no fundo do canal e de velocidades em uma malha tridimensional na bacia central do canal, considerada a bacia de controle. Na sequência, apresenta-se uma breve descrição sobre os equipamentos utilizados nesses ensaios, a metodologia adotada de ensaio e análise dos dados.

\section{Medições de profundidade}

A avaliação da profundidade do escoamento foi realizada utilizando uma ponta limnimétrica fixa em um suporte de madeira móvel sobre o canal, que permite o posicionamento da mesma nos diferentes pontos de medição. As características de ondulação da superfície livre do escoamento exigiram a utilização de um dispositivo auxiliar na definição da superfície d'água média. Foi utilizado junto à ponta limnimétrica um transistor para detectar a resistência da água, mesmo com baixa condutividade, e um LED ("light emitting diode") como sinalizador. A medida da profundidade média do escoamento foi definida, visualmente, a partir do sinalizador mencionado. Considera-se como a medida de profundi- 
dade do escoamento, em cada ponto, aquela obtida na posição em que o sinalizador acende/apaga em intervalos regulares.

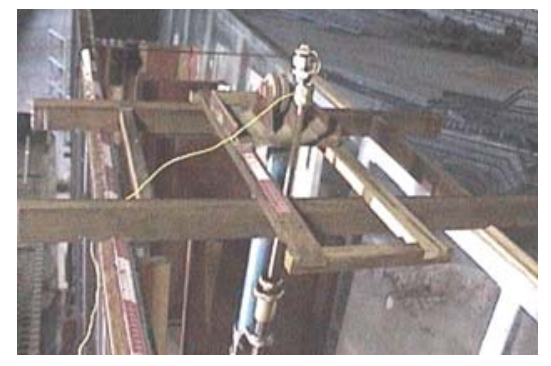

Figura 3 - Ponta limnimétrica fixada ao carrinho móvel sobre o canal.

\section{Pressões}

A medição da pressão no fundo do canal foi realizada através da utilização de transdutores elétricos piezoresistivos de pressão (Figura 4a), para valores instantâneos, e piezômetros convencionais (Figura 4b), para verificação dos valores médios.

Os piezômetros foram construídos com tubos de vidro de $10 \mathrm{~mm}$ de diâmetro, ligados por meio de tubos flexíveis de PVC cristal (diâmetro interno $=1,5 \mathrm{~mm}$; diâmetro externo $=3 \mathrm{~mm}$ ) às tomadas de pressão localizadas no fundo do canal.

Os sensores de pressão utilizados neste trabalho apresentam as seguintes características: marca Hytronic, modelo TM25 com faixa de trabalho de 2 psi ( 1400 mmca), distribuídos da seguinte maneira: $-1,0$ a 1,0 psi; $-0,5$ a 1,5 psi e $-1,5$ a 0,5 psi, com erro de fundo de escala igual a $\pm 0,25 \%$ ( $\pm 3,5$ mm.c.a.).

Todos os transdutores de pressão foram submetidos, periodicamente, a um processo de calibração estática, relacionando medições de tensão a diversas alturas de colunas de água $(\mathrm{P} / \gamma)$.

No total foram distribuídos 175 pontos para as tomadas de pressão. Os ensaios de medição de pressão instantânea foram realizados a uma frequência de aquisição de $50 \mathrm{~Hz}$, com duração de 360 segundos, totalizando 18.000 valores de pressão instantânea para cada tomada de medição. Os ensaios foram realizados em várias campanhas com a utilização de, no máximo, 10 sensores simultaneamente.

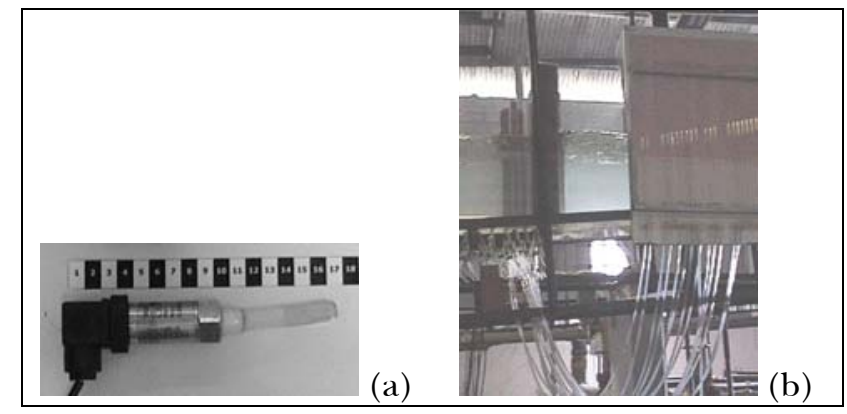

Figura 4 - Medição de pressão: (a) transdutor de pressão sensores piezoresistivos (escala em $\mathrm{cm}$ ); (b) quadro de piezômetros.

\section{Medições de velocidades}

As medições de velocidade foram realizadas com um velocímetro acústico Doppler (ADV) da marca Sontek (Sontek/YSI 16-MHz MicroADV).

As medições de velocidade foram realizadas em uma malha tridimensional na bacia de controle. Realizaram-se medições de velocidades em 5 planos paralelos ao fundo do canal, um deles próximo ao fundo ( $1 \mathrm{~cm}$ do fundo), e os outros afastados do fundo $10 \%, 25 \%, 50 \%$ e $80 \%$ da profundidade média do escoamento $\left(h_{m}\right)$. Em cada um desses planos realizaram-se, no mínimo, medições em 130 pontos (Figura 5a), totalizando 700 pontos de medição na bacia, para cada descarga. Um estudo complementar foi realizado na região da ranhura vertical, sendo esta uma região crítica na passagem dos peixes e, provavelmente, onde ocorre a velocidade máxima. A Figura $5 \mathrm{~b}$ mostra o detalhe em planta da região da ranhura, sendo que ali foram medidas velocidades nas seguintes profundidades (a partir do fundo): $1 \mathrm{~cm}, 10,20,25,30,40,50,60,70$ e $80 \%$ da profundidade média do escoamento. A aquisição dos dados foi realizada utilizando o programa HorizonADV, recomendado pelo fabricante do ADV (HorizonADV, 2005). Durante a realização dos ensaios, foi medida a temperatura da água, sendo esta informada no programa de aquisição de dados. A salinidade não foi medida, sendo considerada igual a zero para todos os ensaios realizados.

Para o posicionamento do equipamento no interior da bacia de medição construiu-se um carrinho móvel sobre o canal que permite ao ADV ser deslocado para qualquer das posições de medição.

O sistema de medição Doppler apresenta um "ruído" que é inerente ao processo. De acordo com recomendações dos fabricantes, devem ser seguidos alguns cuidados durante a realização dos 
Estudo Experimental das Características do Escoamento em Escadas para Peixes do Tipo Ranhura Vertical -

Padrões Gerais do Escoamento

ensaios. O fabricante (Sontek, 2001) alerta que, além da existência de ruído branco no sinal, inerente ao processo de medição, algumas condições do escoamento, como aeração e turbulência elevada, podem prejudicar o sinal, sendo recomendada a aplicação de um filtro às séries de dados.

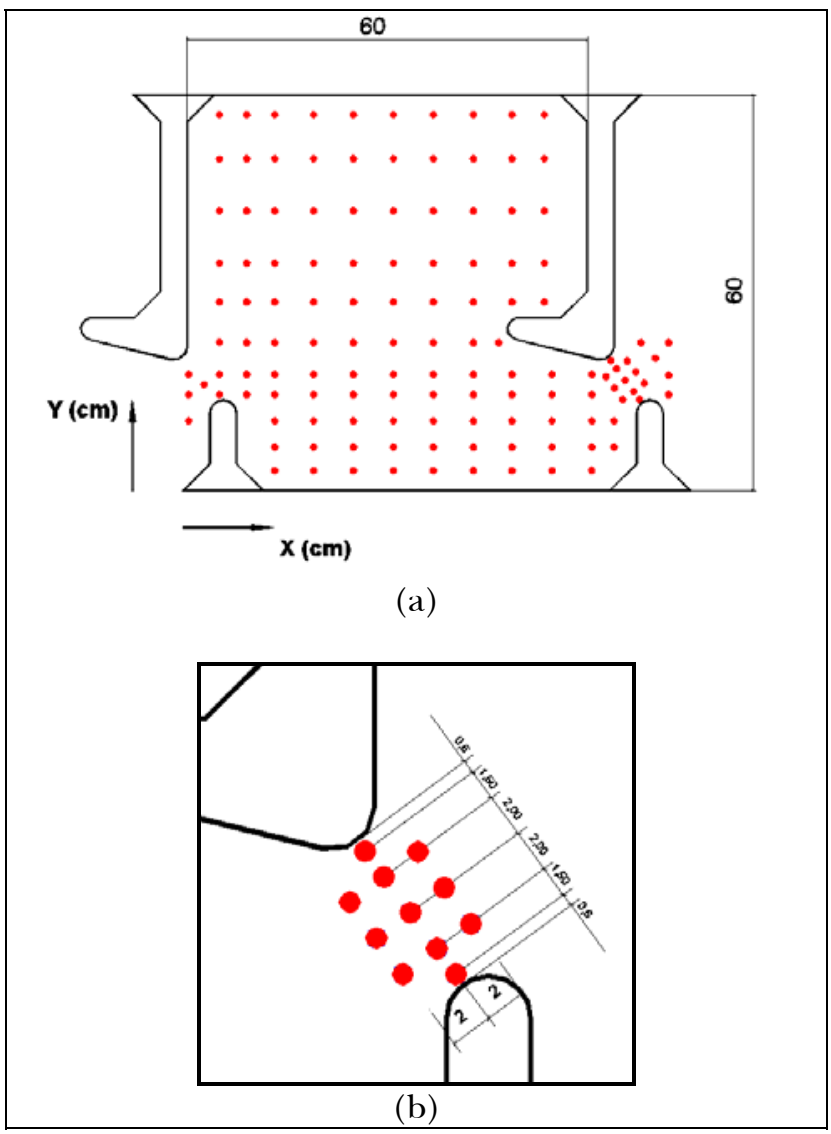

Figura 5 - Pontos de medição de velocidade: (a) na bacia;

(b) detalhe na região da ranhura (dimensões em $\mathrm{cm}$ ).

A partir da avaliação de diferentes filtros, optou-se pela utilização do filtro PSTM (Phase-Space Thresholding Method), desenvolvido por Goring e Nikora (2002) e modificado e implementado no programa WinADV por Wahl (2003).

\section{Condições dos Ensaios}

Foram realizados ensaios para três vazões, de acordo com as condições apresentadas no Quadro 1 . A menor vazão considerada representa a condição de medições realizadas em protótipo, por Viana (2005), e as outras duas descargas representam os valores mínimo e máximo de projeto para a escada da UHE de Igarapava.
Quadro 1 - Resumo das condições do escoamento nas medições realizadas.

\begin{tabular}{lccc}
\hline Condição & 1 & 2 & 3 \\
\hline $\begin{array}{l}\text { Vazão no } \\
\text { modelo } 1: 5 \\
\left(\mathrm{~m}^{3} / \mathrm{s}\right)\end{array}$ & 0,02165 & 0,02451 & 0,02916 \\
\hline $\begin{array}{l}\text { Vazão } \\
\text { correspondente } \\
\text { em protótipo } \\
\left(\mathrm{m}^{3} / \mathrm{s}\right)\end{array}$ & 1,21 & 1,37 & 1,63 \\
\hline $\begin{array}{l}\mathrm{h}_{\mathrm{m}}(\mathrm{m}) \text { no } \\
\text { modelo }\end{array}$ & 0,380 & 0,398 & 0,470 \\
\hline $\begin{array}{l}\Delta \mathrm{h}(\mathrm{m}) \text { no } \\
\text { modelo }\end{array}$ & 0,04 & 0,04 \\
\hline $\begin{array}{l}\mathrm{Fr} * \\
*\end{array}$ & 0,35 & 0,04 \\
\hline $\begin{array}{l}* \mathrm{Fr}=\mathrm{V} / \sqrt{\mathrm{gh}}{ }_{\mathrm{m}} \\
\text { ranhura e e } \mathrm{h}_{\mathrm{m}} \text { é profundidade } \mathrm{V} \text { é a velocidade média do escoamento. }\end{array}$ & 0,35 \\
\hline
\end{tabular}

\section{RESULTADOS E DISCUSSÃO}

\section{Parâmetros hidráulicos}

A seguir são apresentadas as avaliações dos parâmetros hidráulicos em comparação com os dados de outros pesquisadores, obtidos em modelos de escadas para peixes de ranhura vertical semelhantes à avaliada neste estudo.

\section{Coeficiente de Descarga}

A Figura 6 apresenta os coeficientes de descarga obtidos neste estudo, comparando-os com o valor medido na escada da UHE de Igarapava por Viana (2005), os valores obtidos por Coletti (2005) em um modelo dessa estrutura na escala 1:20 e os dados em modelos de Rajaratnam et al. (1986) e (1992). Dos dados de Rajaratnam et al. (1986) e (1992), escolheram-se para comparação os resultados referentes às geometrias 1,16 e 18, que apresentam as configurações mais próximas da avaliada neste estudo. Os dados obtidos por Coletti (2005), Viana (2005) e neste estudo correspondem a ensaios realizados em diferentes escalas de uma mesma estrutura (o modelo utilizado neste estudo é quatro vezes maior que o utilizado por Coletti e cinco vezes menor que o protótipo, da avaliação de Viana). Os pontos correspondentes aos ensaios do presente trabalho encontram-se com a mesma tendência e dispersão dos demais autores, sendo que os valores medidos no modelo e no protótipo, para a mesma 
relação $h_{m} / b_{0}$, indicam o mesmo comportamento em ambas as estruturas.

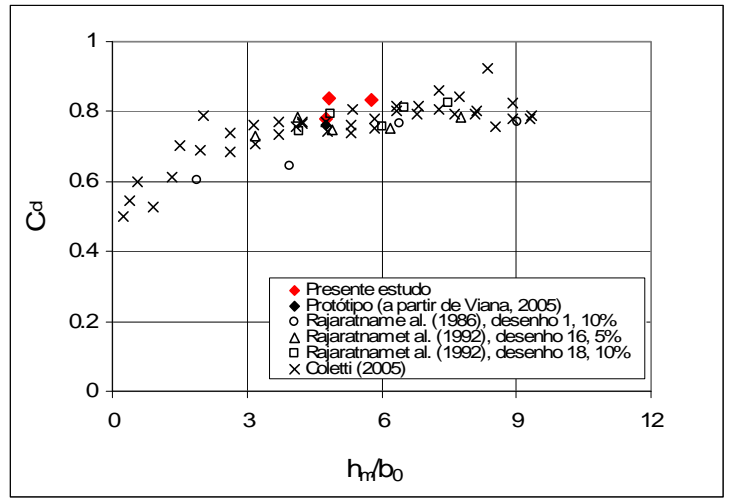

Figura 6 - Coeficientes de descarga obtidos neste estudo comparados com o valor medido em protótipo (Viana, 2005), os dados de Rajaratnam et al. (1986) e Coletti (2005), onde $h_{m}$ é a profundidade média na bacia e $b_{0}$ é a largura da ranhura (na figura declividade das estruturas em percentual).

O valor médio do coeficiente de descarga na avaliação realizada no modelo na escala 1:5 ficou em torno de 0,82 , que é inferior ao valor teórico utilizado no projeto da escada para peixes da UHE de Igarapava, de 0,93, no entanto, a medição em protótipo, por Viana (2005), também aponta valores do coeficiente de descarga inferiores aos considerados no projeto.

\section{Vazão Adimensional}

Os resultados desse parâmetro são apresentados na Figura 7. Além da comparação com o valor medido em protótipo (Viana, 2005) e com os resultados em modelo dos autores Rajaratnam et al. (1986) e (1992) e Coletti (2005), os dados deste estudo foram confrontados com as proposições de Puertas et al. (2004). Puertas et al. (2004) avaliaram duas geometrias de escadas para peixes com ranhura vertical, semelhantes aos desenhos 6 e 16 de Rajaratnam et al. (1992). Os valores encontrados neste trabalho estão entre os diferentes dados/proposições, sendo levemente inferiores à equação proposta por Coletti (2005) e aos dados de Rajaratnam et al. (1986), com boa concordância com o ponto medido no protótipo e um pouco superiores à curva proposta por Puertas et al. (2004), sendo que a geometria denominada T1 é a que mais se aproxima do caso aqui estudado.

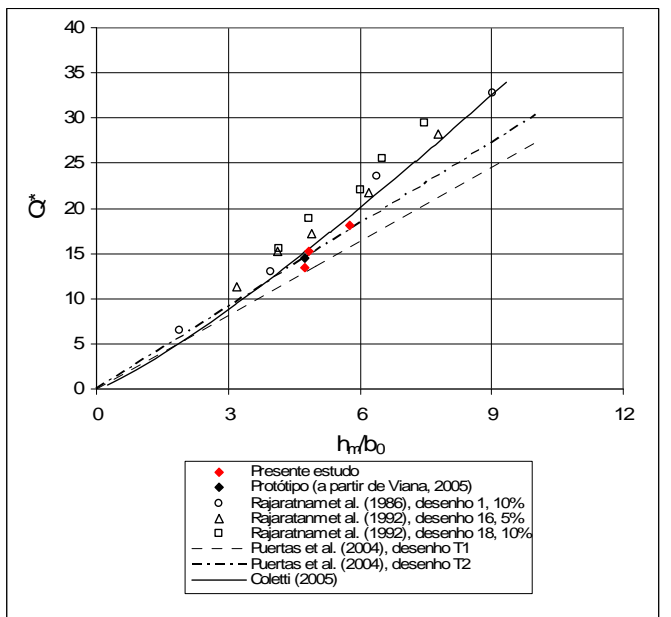

Figura 7 - Vazão adimensional comparada com resultados da literatura.

\section{Coeficiente de Cisalhamento}

Os valores do coeficiente de cisalhamento apresentam-se na Figura 8. Esses valores são levemente superiores aos dados apresentados pelos demais pesquisadores, apresentando uma boa concordância com a medição em protótipo.

Esta análise dos parâmetros hidráulicos: $\mathrm{C}_{\mathrm{d}}$, $\mathrm{Q}^{*}$ e $C f$, para as vazões avaliadas neste estudo, demonstra uma boa concordância com os dados e proposições da literatura. O valor obtido no protótipo, comparado aos parâmetros medidos no modelo, para a mesma relação $h_{m} / b_{0}$, confirma o comportamento semelhante nas duas estruturas.

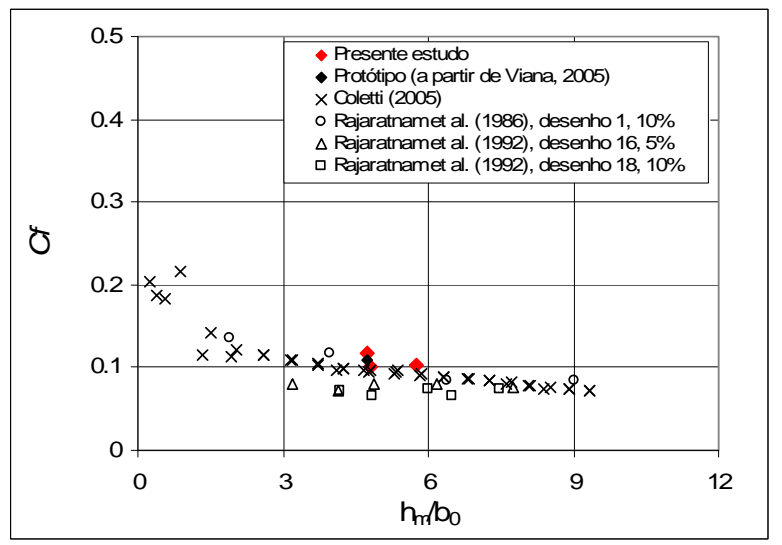

Figura 8 - Coeficiente de cisalhamento comparado com dados da literatura. 


\section{Dissipação de Energia}

A potência dissipada por unidade de volume tem sido utilizada para expressar o grau de agitação do escoamento dentro das bacias das escadas para peixes. Observa-se (tabela 4) que para a estrutura analisada neste trabalho, a potência dissipada por unidade de volume fica entre 138,7 e $151,1 \mathrm{~W} / \mathrm{m}^{3}$ (em protótipo), dentro da faixa recomendada por Bell (1973) apud Bell (1990), onde coloca que esse valor não deve exceder $191 \mathrm{~W} / \mathrm{m}$.

Tabela 4 - Principais características das descargas testadas

\begin{tabular}{llll}
\hline \multicolumn{4}{l}{ Modelo Escala 1:5 (Protótipo 1:1) } \\
\hline $\mathrm{Q}\left(\mathrm{m}^{3} / \mathrm{s}\right)$ & $\mathrm{h}_{\mathrm{m}}(\mathrm{m})$ & $\Delta \mathrm{h}(\mathrm{m})$ & $\mathrm{P}_{\mathrm{V}}\left(\mathrm{W} / \mathrm{m}^{3}\right)$ \\
\hline 0,02165 & 0,380 & 0,04 & 62,04 \\
$(1,21)$ & $(1,90)$ & $(0,20)$ & $(138,7)$ \\
0,02451 & 0,398 & 0,04 & 67,06 \\
$(1,37)$ & $(1,99)$ & $(0,20)$ & $(149,9)$ \\
0,02916 & 0,470 & 0,04 & 67,56 \\
$(1,63)$ & $(2,35)$ & $(0,20)$ & $(151,1)$ \\
\hline
\end{tabular}

\section{Profundidade do Escoamento e Pressões}

A avaliação da profundidade do escoamento pode ser obtida a partir das medições na superfície do escoamento, utilizando a ponta limnimétrica ou, indiretamente, através das pressões médias. A Figura 9a mostra os resultados de pressão média distribuídos dentro da bacia de controle. Observou-se, para as diferentes descargas, o mesmo tipo de comportamento quanto às profundidades do escoamento dentro da bacia. A profundidade do escoamento é maior na parte de montante do septo maior e a montante da ranhura, na região de aproximação. Ao passar pela ranhura, observa-se o rebaixamento da superfície d'água de forma acentuada, como esperado, já que há o aumento da velocidade nessa região. Avaliando as seções transversais à bacia, temse que na região do jato principal as profundidades são menores em relação às regiões de recirculação. Observando-se o comportamento da superfície livre obtido por Pena (2004), para declividades do canal de $5,7 \%$ e $10,0 \%$, verifica-se que, mesmo com geometrias diferentes, as características gerais são semelhantes as desta estrutura.

A seguir apresentam-se os resultados obtidos para a vazão correspondente à estudada por Coletti (2005) em um modelo na escala 1:20, quatro vezes menor que o aqui avaliado (escala 1:5). A amplitude das pressões médias obtidas neste estudo é inferior à observada por Coletti (2005), obedecendo às leis de semelhança adequadas. Coletti (2005) verificou no campo de pressões médias a formação, na região da recirculação maior, de duas zonas diferentes: uma com valores de pressão maiores que a média e outra com valores inferiores, o que não foi encontrado neste trabalho. A região a montante da ranhura apresenta valores de pressão maiores, que diminuem bruscamente com a passagem pela abertura, observado neste trabalho e por Coletti (2005), seguindo o comportamento das variações da profundidade do escoamento.

As Figuras 9b, 9c e 9d apresentam a variação, no interior da bacia, de alguns momentos de distribuição estatística obtidos a partir das séries temporais de pressão. Observa-se, como encontrado por Coletti (2005), que a região do jato apresenta valores de desvio padrão maiores que os presentes nas regiões de recirculação. Analisando os campos dos coeficientes de assimetria e curtose, observa-se que é na região do jato que os valores se afastam mais do comportamento característico de uma distribuição normal.

\section{Velocidades Médias}

A avaliação dos campos de velocidades médias é fundamental para a definição das características principais do escoamento. A visualização do campo de velocidades médias auxilia na definição do padrão do escoamento dentro da bacia e na caracterização das magnitudes e distribuição dos vetores velocidade. Nessa fase podem ser identificados determinados comportamentos do fluxo, como regiões de recirculação, zonas com velocidades elevadas, entre outros, que dificultam ou impossibilitam a utilização da escada para peixes por certos indivíduos.

A Figura 10 apresenta os campos de velocidades em planos paralelos ao fundo do canal para uma das descargas ensaiadas. Os vetores indicam as componentes em planos horizontais e em escalas de cores são representadas as componentes verticais de velocidade. Nas três vazões ensaiadas para as diferentes profundidades do escoamento observa-se a formação de um jato principal, caracterizado pelo fluxo entre ranhuras consecutivas, onde as velocidades são maiores, conforme já observado em estudos anteriores em escadas para peixes do tipo ranhura vertical: Rajaratnam et al., (1986) e (1992), Wu et al. (1999), Pena (2004), Liu et al. (2006), Liu (2004), 
Viana (2005), entre outros. Nas regiões adjacentes ao jato principal formam-se zonas de recirculação: uma maior, confinada pelos defletores maiores, pela parede lateral e pelo jato principal, e outra recirculação menor, formada do outro lado, entre os defletores menores, o jato principal e a parede lateral oposta.

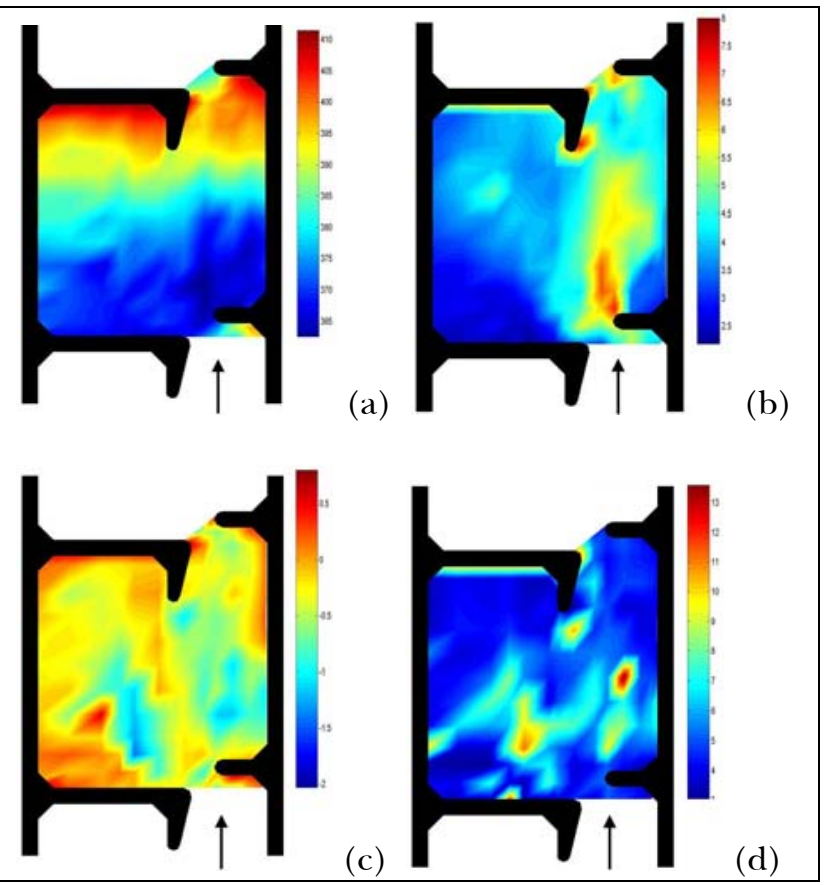

Figura 9 - Séries temporais de pressão no fundo do canal: (a) pressões médias; (b) desvio padrão; (c) coeficiente de assimetria; (d) coeficiente de curtose, ao longo da bacia de controle para a vazão de $0,02165 \mathrm{~m}^{3} / \mathrm{s}$ em modelo na escala 1:5.

Na Figura 10, as componentes verticais da velocidade estão representadas através dos mapas de cores. Observa-se que estas são de pequena magnitude em comparação com as componentes horizontais presentes nos planos paralelos ao fundo do canal. Verifica-se que as maiores componentes verticais encontram-se em planos mais superficiais e em duas regiões: logo após a passagem da água pela ranhura e junto ao defletor maior de jusante. Nos dois casos as componentes são positivas, indicando um fluxo ascensional. No primeiro caso, têm-se efeitos devido à passagem de água pela ranhura. No segundo caso, tem-se uma sobreelevação do escoamento devido ao efeito de represamento causado pelo defletor. Observam-se algumas componentes de velocidades verticais negativas em pontos isola- dos, que, a princípio, não definem um comportamento padrão.

As variações das componentes de velocidade vertical estão entre $-0,30 \mathrm{~m} / \mathrm{s}$ e $+0,20 \mathrm{~m} / \mathrm{s}$. Comparando-se esses valores com os valores máximos dos vetores de velocidade média nos planos horizontais, que estão na ordem de $1,00 \mathrm{~m} / \mathrm{s}$, considera-se que as componentes verticais são pouco significativas.

Para verificar a variação dos campos de velocidade em diferentes planos paralelos ao fundo, avaliou-se o comportamento dos vetores de velocidade para as diferentes profundidades no caminho do jato principal, conforme pode ser observado na Figura 11. Nessa figura tem-se que as componentes de velocidade nos planos paralelos à soleira apresentam, nas três vazões ensaiadas, comportamento praticamente independente da profundidade do escoamento no caminho do jato principal. Observa-se que ao serem comparadas as magnitudes dos vetores de velocidade das três vazões têm-se valores independentes da descarga. Confirma-se que o valor da velocidade em determinado ponto da bacia é independente da profundidade e da vazão, conforme já foi relatado por Pena (2004) em escadas do tipo ranhura vertical com geometrias diferentes da que está sendo aqui avaliada.

A região da ranhura representa um ponto crítico na transposição dos peixes pela estrutura por dois motivos: primeiro, porque representa um ponto de passagem obrigatória e segundo, porque nesta região, provavelmente, encontram-se as máximas velocidades. Optou-se em mostrar esses dados na forma de campos de velocidade, em um plano vertical que une os dois septos (Figura 5b), conforme apresentado na Figura 12a para uma das vazões. Nessa figura o mapa de cores indica as componentes horizontais das velocidades na ranhura. Observa-se, para as três vazões, uma região de velocidades mais elevadas junto ao defletor menor, onde o jato principal tem a entrada direta. Próximo à parte interna da ranhura (junto ao septo maior) têm-se velocidades menores, provavelmente porque o escoamento que vem do jato principal perde energia por efeito cisalhante junto ao septo maior, enquanto que o septo menor entra em contato com o jato somente quando este já está passando pela abertura. Observase que as máximas componentes ocorrem junto ao fundo e na profundidade correspondente a $10 \%$ da altura média do escoamento. Analisando as componentes verticais (Figura 12b) observa-se que, na maior parte, são desconsideráveis (valores entre $0,10 \mathrm{~m} / \mathrm{s} \mathrm{e}+0,10 \mathrm{~m} / \mathrm{s}$ ), caracterizando um jato praticamente bidimensional na passagem pela abertura. 
Estudo Experimental das Características do Escoamento em Escadas para Peixes do Tipo Ranhura Vertical Padrões Gerais do Escoamento
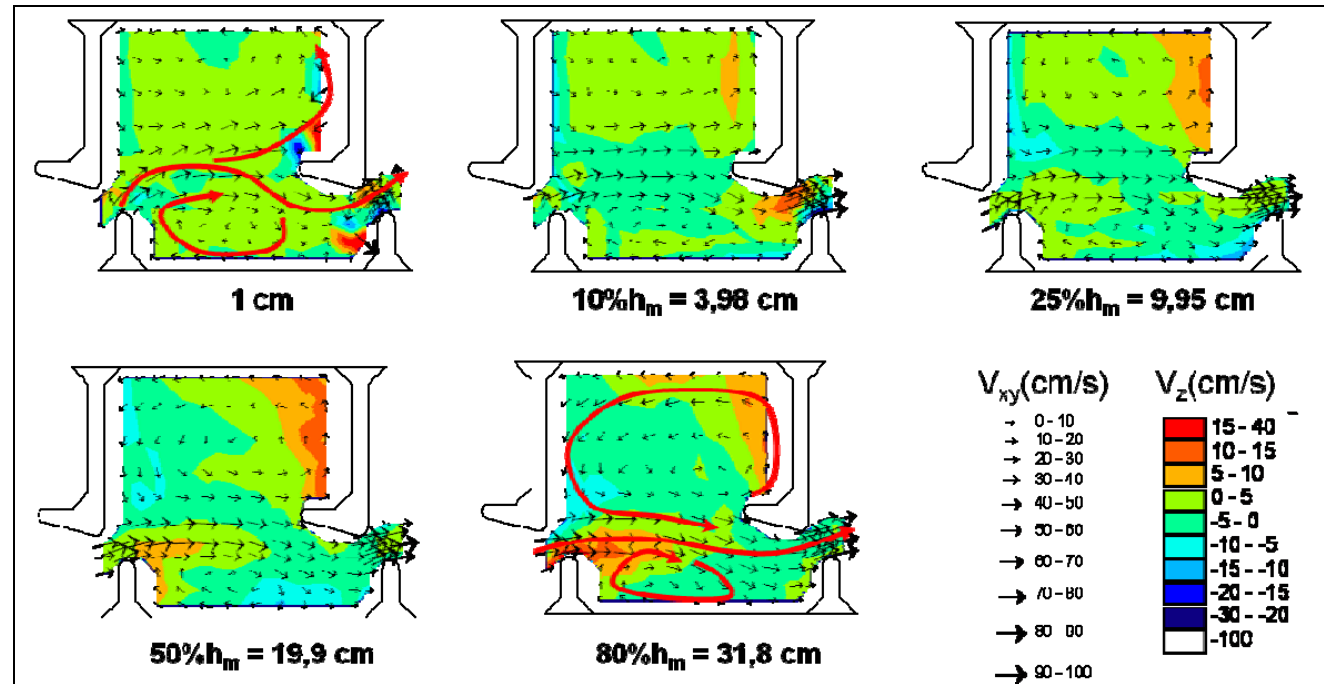

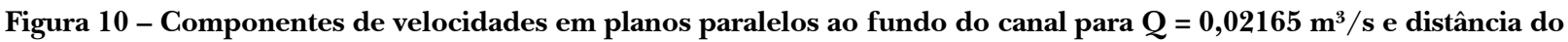
fundo ao plano (h): (a) $h=1 \mathrm{~cm} ;(\mathrm{b}) \mathrm{h}=0.10 \mathrm{hm} ;$ (c) $h=0.25 \mathrm{hm} ;$ (d) $h=0.50 \mathrm{hm} \mathrm{e}(\mathrm{e}) \mathrm{h}=0.80 \mathrm{hm}$.

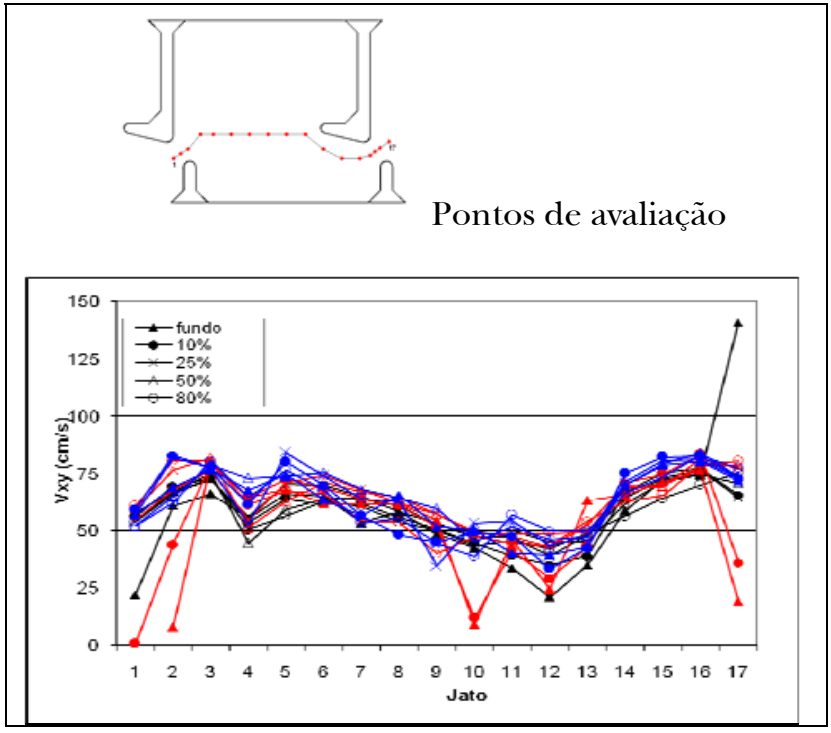

Figura 11 - Velocidades no plano paralelo ao fundo, em diferentes profundidades, na linha do jato principal para: $Q=0,02165 \mathrm{~m}^{3} / \mathrm{s}$ (preto); $Q=0,02451 \mathrm{~m}^{3} / \mathrm{s}$ (vermelho) e $\mathrm{Q}=0,02916 \mathrm{~m}^{3} / \mathrm{s}$ (azul).

A Figura 13 mostra as velocidades na região da ranhura na forma de perfis de velocidade. Observou-se que a máxima velocidade na ranhura ocorre próximo ao fundo, como observado por Wu et al. (1999). Neste estudo a máxima velocidade média medida foi de $0,92 \mathrm{~m} / \mathrm{s}$, que se aproxima da velocidade potencial, $(2 \mathrm{~g} \Delta \mathrm{h})^{0,5}=0,89 \mathrm{~m} / \mathrm{s}$ (onde $\Delta \mathrm{h}$ é a carga hidráulica entre bacias consecutivas, que para as condições de ensaio é igual a 0,04 m). Conside- rando os critérios de semelhança de Froude, a máxima velocidade no protótipo é aproximadamente $2 \mathrm{~m} / \mathrm{s}$.

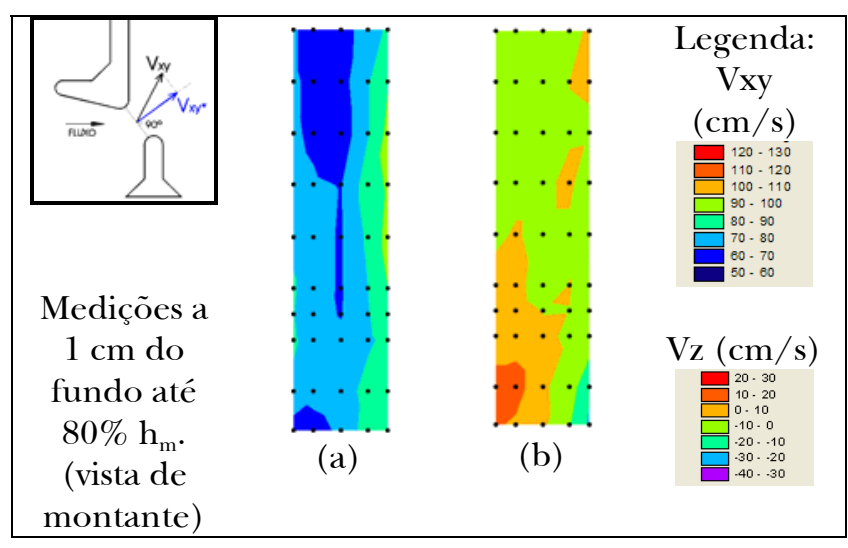

Figura 12 - Velocidades na ranhura para $Q=0,02451 \mathrm{~m}^{3} / \mathrm{s}$ : (a) $V_{x y}$ e (b) $V_{z}$

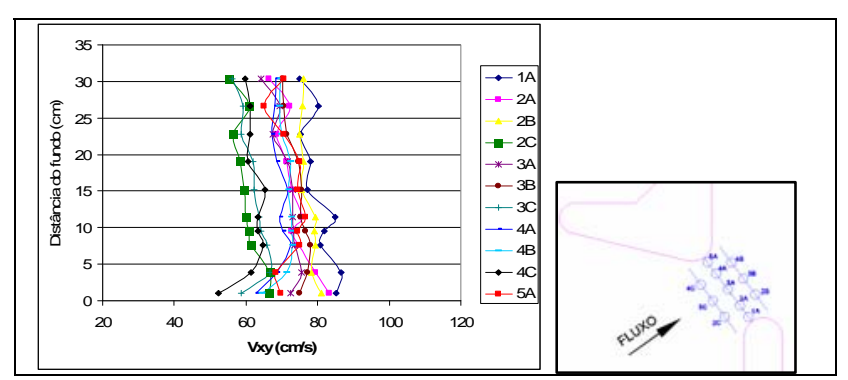

Figura 13 - Perfis de velocidades resultantes horizontais na ranhura $\left(V_{x y}\right)$ para $Q=0,02165 \mathrm{~m}^{3} / \mathrm{s}$. 
Na Figura 14 comparam-se os resultados obtidos neste estudo (escala 1:5) com os valores obtidos por Viana (2005) em medições no protótipo e em um modelo na escala 1:20. São comparadas as máximas velocidades médias medidas na região da ranhura para diferentes profundidades. As medições de Viana (2005) foram realizadas no modelo 1:20 com anemometria Laser Doppler e no protótipo com um molinete. Todos os resultados foram transpostos para a escala do protótipo.

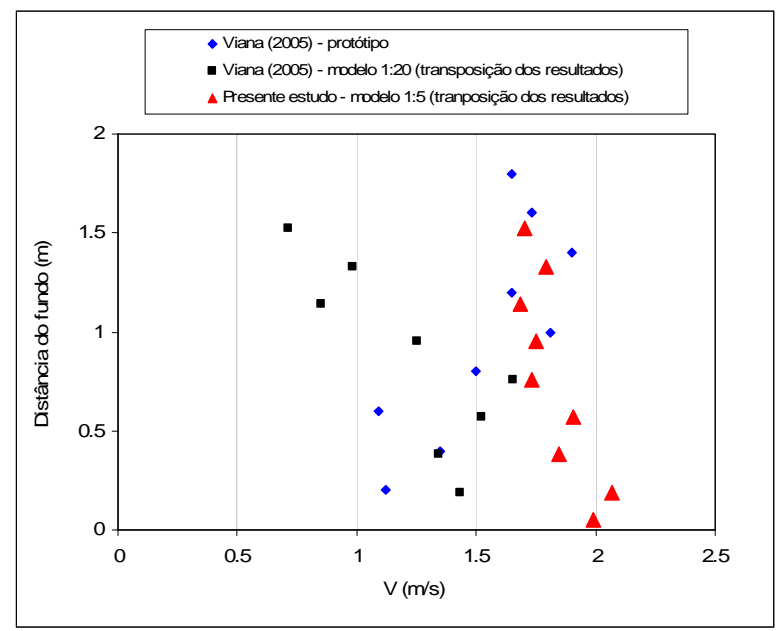

Figura 14 - Máximas velocidades médias na seção da ranhura para $Q=1,21 \mathrm{~m}^{3} / \mathrm{s}$ (em protótipo).

Observa-se que os valores máximos de velocidade média na seção da ranhura obtidos no modelo 1:5 e os medidos em protótipo são semelhantes para a metade superior do perfil de velocidades. $\mathrm{Na}$ metade inferior, verifica-se que as medições realizadas no modelo na escala 1:5 são superiores aos valores do protótipo e do modelo 1:20. Essas diferenças podem ser resultantes de diferentes causas, entre elas: efeito de escala (protótipo, escala 1:5 e escala 1:20); medições com diferentes tipos de aparelhos (no protótipo foi utilizado um fluxímetro, no modelo na escala 1:5 um ADV e na escala 1:20 um anemômetro a laser); diferenças na posição dos pontos de medição e presença de pedras ou outros obstáculos no fundo do canal no protótipo.

Neste estudo, notou-se que não ocorrem variações significativas das componentes de velocidade para diferentes profundidades em uma mesma posição da bacia, não somente na seção da ranhura, mas nas demais regiões.

No presente trabalho, as máximas velocidades médias ocorreram na seção da ranhura, diferen- te do observado por Viana (2005), onde os máximos valores médios encontraram-se dentro da bacia.

\section{Ictiofauna x Características Hidráulicas}

$\mathrm{Na}$ escada de ranhura vertical a seção de comunicação entre bacias consecutivas representa um ponto de passagem obrigatório onde ocorrem as maiores velocidades. Considerando os tipos de velocidades características dos peixes, tem-se como limitante para a passagem pela ranhura, que a velocidade de explosão da espécie seja maior do que a velocidade encontrada na ranhura para que o mesmo tenha chances de realizar a transposição, mesmo com um esforço excessivo, que não é o desejado. $\mathrm{O}$ que seria mais razoável admitir seria a consideração da velocidade máxima na ranhura inferior à velocidade crítica das espécies. Ultrapassando o obstáculo das maiores velocidades dentro de cada bacia, os indivíduos encontram regiões de descanso onde, se necessário, podem se recuperar entre as passagens pelas ranhuras.

Complementando esse estudo, em uma avaliação qualitativa, foram inseridos peixes adultos (Astyanax bimaculatus) de dimensões compatíveis com o modelo na escala 1:20 (ranhura de $2 \mathrm{~cm}$ e 27 bacias de 0,15 x 0,15 m, modelo descrito em Coletti, 2005), sendo registrada a trajetória do mesmo através de câmara digital. De uma maneira geral, observa-se um comportamento padrão durante o deslocamento do peixe para montante. O mesmo fica posicionado entre o jato principal e a recirculação maior, escolhendo passar pela ranhura junto ao defletor maior. Observou-se, também, através da análise de vários indivíduos que, geralmente, a "negociação" para a passagem pela primeira abertura leva mais tempo e então, nas demais passagens, o tempo é reduzido. Ressalta-se que estas avaliações representam uma análise meramente qualitativa e reforça-se a necessidade de estudos que considerem as diversas componentes biológicas envolvidas.

Os campos de velocidade do escoamento devem ser comparados com as características natatórias das espécies que utilizam este MTP. Santos (2007) avaliou a velocidade crítica de três espécies de peixes que utilizam a escada para peixes da UHE Igarapava. As três espécies testadas por Santos (2007) foram o mandi (Pimelodus maculatus), o piau (Leporinus reinhardti) e o curimba (Prochilodus costatus), cujas velocidades críticas foram avaliadas em $1,47,1,32$ e 1,23 m/s, respectivamente.

A partir desses valores e considerando que essas espécies de peixes são observadas na passagem pela escada para peixes da UHE de Igarapava, op- 
tou-se em confrontar os valores de velocidade crítica avaliados por Santos (2007), com os campos de velocidades médias medidas em modelo, realizando as devidas transposições de escala (1:5).

A Figura 15 apresenta, para um dos campos de velocidade, as regiões cuja velocidade excede a velocidade crítica desses peixes. Nessas figuras delimitam-se as regiões em verde, que representam zonas com velocidades superiores à velocidade crítica do curimba $(>1,23 \mathrm{~m} / \mathrm{s})$; em amarelo, as velocidades superiores à velocidade crítica do curimba e do piau $(>1,32 \mathrm{~m} / \mathrm{s})$ e em vermelho, maiores que à velocidade crítica das três espécies $(>1,47 \mathrm{~m} / \mathrm{s})$. Verifica-se que na região da ranhura e a jusante desta, no caminho do jato principal, encontram-se valores de velocidade superiores à velocidade crítica dessas três espécies. A barreira imposta em parte do caminho do jato principal foi observada durante o estudo da trajetória dos indivíduos. Observou-se, claramente, que os indivíduos preferem a aproximação da ranhura pela região da bacia adjacente à recirculação maior, assim desviando das regiões com maiores velocidades, que formam uma barreira hidráulica. Verifica-se que em praticamente toda a seção da ranhura, as velocidades são superiores às velocidades críticas das espécies avaliadas. Esse fato indica que os peixes dessas espécies utilizam velocidades superiores à velocidade crítica para passar de uma bacia a outra.

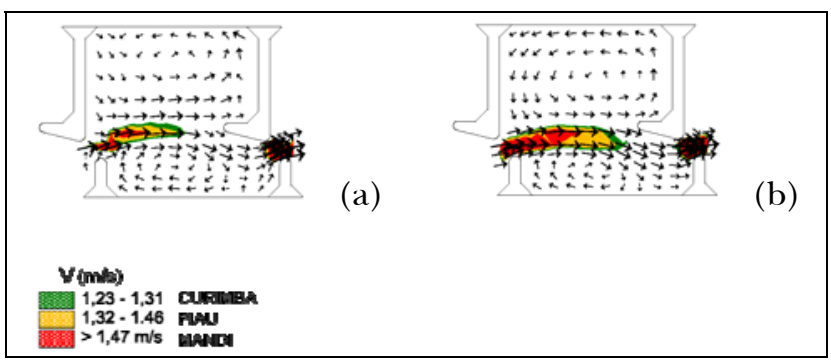

Figura 15 - Delimitação das regiões com velocidades superiores à velocidade crítica das espécies mandi $(1,47 \mathrm{~m} / \mathrm{s})$, piau $(1,32 \mathrm{~m} / \mathrm{s})$ e curimba $(1,23 \mathrm{~m} / \mathrm{s})$ para $\mathrm{Q}=1,37 \mathrm{~m}^{3} / \mathrm{s}$ (em protótipo), em planos paralelos ao fundo: (a) $10 \% \mathrm{~h}_{\mathrm{m}}$ e (b) $50 \% \mathrm{~h}_{\mathrm{m}}$. (distâncias em relação ao fundo do canal).

\section{CONCLUSÕES}

Apresentaram-se neste trabalho resultados de um estudo experimental realizado em um modelo físico de uma escada para peixes do tipo ranhura vertical. A avaliação dos parâmetros hidráulicos do escoamento: coeficiente de descarga, vazão adimensional e coeficiente de cisalhamento, demonstraram que o escoamento nessa estrutura apresenta o comportamento semelhante ao relatado na bibliografia. A geometria utilizada nessse estudo é idêntica a de Viana (2005) e Coletti (2005), diferenciando-se apenas pela escala do modelo, com relação comprimento/largura da bacia igual a 1,0. Já as geometrias analisadas por Rajaratnam et al. (1986) e (1992) e Puertas et al. (2004)apresentavam relação comprimento/largura da bacia de 1,25. Essa pequena variação nas geometrias não demonstrou interferir na avaliação dos parâmetros hidráulicos. Salientase que a abertura da ranhura apresenta-se proporcional em todas as geometrias analisadas.

Os mapas de níveis de água da superfície livre do escoamento e de pressões mostram um comportamento semelhante para as diferentes descargas, caracterizado pela maior profundidade do escoamento na parte de montante do septo maior, e a montante da ranhura, na região de aproximação da mesma. A análise das flutuações de pressão apontou que, na região do jato, os valores de desvio padrão são maiores que os presentes nas regiões de recirculação. Analisando os campos dos coeficientes de assimetria e curtose, tem-se que é no jato principal que os valores se afastam mais do comportamento característico de uma distribuição normal.

A análise das velocidades médias do escoamento na escada com ranhura vertical indicou o comportamento geral do escoamento de acordo com o encontrado em estudos anteriores: tem-se um jato principal conectando ranhuras consecutivas, onde as velocidades são as mais elevadas dentro das bacias, com componentes predominantemente horizontais; adjacentes ao jato principal existem duas zonas de recirculação do escoamento, uma de cada lado, onde as velocidades são menores. Os campos de velocidades nos planos paralelos à soleira indicam não existirem variações significativas do comportamento do escoamento ao longo da profundidade. Ao serem comparadas as magnitudes dos vetores de velocidade das três descargas testadas, para uma mesma posição na bacia, observam-se valores independentes da vazão.

A comparação da caracterização do escoamento com o comportamento dos peixes indica que a trajetória preferencial do mesmo, no caminho dentro da bacia, coincide com condições favoráveis sob o ponto de vista hidráulico. Observou-se, no estudo de Viana (2005), onde foram testados peixes em um modelo na escala 1:10, e em uma avaliação qualitativa realizada neste trabalho, onde foram 
testados peixes em um modelo na escala 1:20, que os peixes preferem se deslocar adjacentes ao jato principal, do lado da recirculação maior. Na passagem pela ranhura, o mesmo prefere a aproximação junto ao defletor maior, onde foram observadas menores velocidades. No entanto, salienta-se a necessidade de estudos que considerem as diversas componentes biológicas envolvidas e que possam fornecer resultados quantitativos, além de qualitativos.

Conclui-se ainda, que o uso de modelos físicos tem demonstrado ser uma ferramenta muito valiosa na avaliação do escoamento em escadas para peixes, principalmente pela possibilidade de análise comparativa entre diferentes geometrias. Os parâmetros hidráulicos e campos de velocidades obtidos podem ser utilizados na seleção de estruturas mais adequadas, com base na comparação dos resultados observados com estudos que consideram o comportamento biológico das espécies face esses parâmetros caracterizadores.

\section{AGRADECIMENTOS}

Os autores agradecem o apoio da CAPES, pela concessão da bolsa de estudos de doutorado da primeira autora e ao $\mathrm{CNPq}$ pelo apoio no projeto.

\section{REFERÊNCIAS}

BELL, M. C. (1973). Fisheries Handbook of Engineering Requirements and Biological Criteria, U. S. Army Corps of Engineers, North Pacific Division, Portland, Oregon.

CBDB - Comitê Brasileiro de Barragens. Barragens e Peixes Análise crítica e recomendações (1999). Boletim 116, 115p.

CLAY, C.H. (1995). Design of fishways and other fish facilities. (2nd ed). Boca Raton, Lewis, 248p.

COLETTI, J.Z. (2005). Características do escoamento ao longo de uma escada de peixes do tipo ranhura vertical, Dissertação de mestrado, Programa de PósGraduação em Recursos Hídricos e Saneamento Ambiental, IPH/UFRGS.

GORING, D.G.; NIKORA, V.I. (2002). "Despiking Acoustic Doppler Velocimeter Data". Journal of Hydraulic Engineering, ASCE, Vol. 128, No. 1, 117-126.

LARINIER, M. Pool fishways, pre-barrages and natural bypass channels. In: Fishways: biological basis, design crite- ria and monitoring, Bull. FR. Pêche Piscic. n³64, 5482, 2002.

LIU, M. Turbulence Structure in Hydraulics Jumps and Vertical Slot Fishways. Thesis, University of Alberta, 313 p., 2004.

LIU, M.; RAJARATNAM, N., ZHU, D.Z. (2006). "Mean Flow and Turbulence Structure in Vertical Slot Fishways". Journal of Hydraulic Engineering, v. 132, n. 8, p. 765777.

LUPANDIN, A.I. (2005). Effect of flow turbulence on swimming speed of fish. Biology Bulletin, 32(5): 461-466. Translated from Izvestiya Akademii Nauk, Seriya Biologicheskaya, 5:558-565.

ODEH, M. et al. U.S. Geological Survey, Glenn F. Cada - Oak Ridge National Laboratory, 2002, Evaluation of the Effects of Turbulence on the Behavior of Migratory Fish, Final Report 2002, Report to Bonneville Power Administration, Contract No. 00000022 , Project No. 200005700, 55 electronic pages (BPA Report DOE/BP-00000022-1)

PENA, L. (2004). Estudio hidráulico en modelo de escalas de peixes de fenda vertical e de fenda profunda aliñadas. Aproximación á avaliación experimental da enerxía cinética turbulenta. Tese de Doutoramento, Programa de Doutoramento de Enxeñería Civil, Universidade da Coruña.

PUERTAS, J.; PENA, L.; TEIJEIRO, T. (2004). "Experimental approach to the hydraulic of vertical slot". Journal of Hydraulic Engineering, ASCE, Vol. 130, No. 1, 1-14, 2004.

RAJARATNAM, N.; KATOPODIS, C. (1986). "Hydraulics of vertical slot fishways". Journal of Hydraulic Engineering, ASCE, Vol. 112, No. 10, 909-927.

RAJARATNAM, N., KATOPODIS, C., SOLANKI, S. (1992). "New design for vertical slot fishways". Canadian Journal of Civil Engineering. Volume 19, Número 3, 402-414.

SANTOS, H.A. (2007). A Influência da Capacidade Natatória de Peixes Neotropicais no Projeto Hidráulico de Mecanismos de Transposição, Tese de Doutorado, Programa de Pós Graduação em Saneamento, Meio Ambiente e Recursos Hídricos, UFMG.

SONTEK. (2001). Sontek ADVField Acoustic Doppler Velocimeter. Technical Documentation. San Diego, USA.

VIANA, E.M.F. (2005). Mapeamento do Campo de Velocidades em Mecanismos de Transposição de Peixes do Tipo Slot Vertical em Diferentes Escalas, Tese de Doutorado, Programa de Pós Graduação em Saneamento, Meio Ambiente e Recursos Hídricos, UFMG.

WAHL, T.L. (2003). Discussion of "Despiking Acoustic Doppler Velocimeter Data" by D. G. Goring \& V. I. Nikora. Journal of Hydraulic Engineering, ASCE, Vol. 129, No. 6, 484-487. 
WU, S.; RAJARATNAM, N.; KATOPODIS, C. (1999). "Structure of flow in vertical slot fishway". Journal of Hydraulic Engineering, ASCE, Vol. 125, No. 4, 351-360.

\section{Experimental Study of the Flow Characteristics in Vertical Slot Fishways - General Flow Patterns}

\section{ABSTRACT}

Dam construction along the rivers affects the biological communities by changing aquatic habitats, interfering in fish movements and causing serious restrictions to the reproduction of migratory species. In an attempt to reduce these effects, many dams include fishways to help species maintain their life cycles. Fishways can be of various types according to the obstacle height, fish species and available discharges. In this paper, flow patterns in a vertical slot fishway were studied. An experimental arrangement consisting of a $0.60 \mathrm{~m}$ wide, $0.60 \mathrm{~m}$ deep and 9 $m$ long flume divided into nine pools was evaluated. This model represents a part of the fishway installed at the Igarapava Hydropower Plant in Minas Gerais (Brazil). Measurements were carried out on the flow depth, bottom pressure and flow velocity in one of the pools for three discharges. The slot region between consecutive pools was studied in detail since it represents an obligatory passage and it is where the highest velocities are expected. The results of this paper were divided in two parts: (1) the average flow parameters and (2) the characteristics of the turbulent flow. This paper (part (1) of the study) presents the non-dimensional flow parameters and isoline maps showing the characteristics of the flow depth, pressures and mean velocities. Through the analysis of results it was possible to characterize the average behavior of the flow. The results allowed defining important non-dimensional parameters of these structures, such as the discharge coefficient and the non-dimensional flow rate. Flow velocities do not change based on discharge and flow depth, the pool position is the main factor of interference. The maximum velocity of the flow can reach up to $2.0 \mathrm{~m} / \mathrm{s}$ (prototype scale) and the horizontal components are predominant. The vertical components do not exceed $0.7 \mathrm{~m} / \mathrm{s}$ (prototype scale) in isolated regions. An analysis that contrasted the velocity fields of the structure and the velocity characteristics of some species of Brazilian ichthyofauna demonstrated that there are regions of the flow with barriers preventing the free passage of these species.

Key-words: fishway, fish passes, velocities, hydraulic parameters, energy dissipation. 